\title{
Search for the Standard Model Higgs boson decaying to b-quark pairs with the ATLAS detector at the LHC
}

\section{P. Conde Muíño* on behalf of the ATLAS Collaboration}

LIP (Laboratório de Instrumentação e Física Experimental de Partículas), Elias Garcia 14, 1000-149 Lisbon, PT and

Departamento de Física, Faculdade de Ciências, Universidade de Lisboa, 1749-016 Lisbon, PT

E-mail: pconde@lip.pt

\begin{abstract}
After many years of searches, the Higgs boson was observed by the ATLAS and CMS collaborations in July 2012. Since then, many of its properties have been measured using primarily the bosonic decay channels: $H \rightarrow \gamma \gamma, H \rightarrow W^{+} W^{-} \rightarrow l v l v$ and $H \rightarrow Z Z \rightarrow l l l^{\prime} l^{\prime}$. In order to probe the predictions of the Standard Model and the nature of the Higgs boson it is also fundamental to measure its couplings to fermions and, in particular, to quarks. In this paper we present the ATLAS results in the search for the Higgs boson decaying to b-quark pairs using two different production channels: associated production with a vector boson and vector boson fusion with an additional hard photon, using approximately $13 \mathrm{fb}^{-1}$ of pp collisions delivered by the LHC at a center of mass energy of $13 \mathrm{TeV}$.
\end{abstract}

38th International Conference on High Energy Physics

3-10 August 2016

Chicago, USA

${ }^{*}$ Speaker. 


\section{Introduction}

Since its discovery [1] in 2012, the Higgs boson properties have been measured using mainly bosonic decay channels. The results show that the observed particle is compatible with the Standard Model (SM) Higgs boson within the accuracy of the measurements [2]. With respect to fermionic decay modes, the first observation of decays to $\tau$-pairs was obtained with the combination of ATLAS and CMS Run 1 data [2], while for the decays to b-quark pairs the combined significance does not yet reach the $3 \sigma$ evidence level. Having the largest branching fraction in the $\mathrm{SM}, B R(H \rightarrow b \bar{b}) \simeq 58 \%$, the observation of the Higgs decaying to b-quark pairs is also essential to constraint the total Higgs width.

The ATLAS experiment [3] has searched for the Higgs decays to b-quark pairs using $\sim 13 \mathrm{fb}^{-1}$ of proton-proton collisions collected during the LHC Run 2, with a center of mass energy of $13 \mathrm{TeV}$. Given the huge background from b-quark pairs produced directly in the pp collisions, the search was done using two different sub-dominant production modes: associated production with a $W$ or a $Z$ boson (called together VH production) and vector boson fusion (VBF) with a high-p $\mathrm{p}_{\mathrm{T}}$ photon accompanying the Higgs. Despite the reduction in the signal cross section due to the presence of the hard photon in the VBF channel, the dominant background $b b+\gamma+$ jet jet is suppressed by a factor $\sim 10$, leading to an improved signal over background ratio. In what follows, these two searches will be explained in more detail.

\section{Search for $H \rightarrow b b$ in associated production with a vector boson}

The search for the Higgs boson in this channel has been done considering the leptonic decays of the $W$ and $Z$ boson, which result in three different final states: $W H \rightarrow l v b \bar{b}, Z H \rightarrow l l b \bar{b}$ and $Z H \rightarrow v \bar{v} b \bar{b}$. These channels are characterized by one, two or zero charged leptons in the final state, respectively. The experimental signatures, in addition to the two b-jets, are therefore high transverse momentum $\left(p_{T}\right)$ isolated charged leptons ${ }^{1}$ or large missing transverse energy, that are also used to trigger the event. The selection benefited from better b-tagging with respect to the Run 1 analysis, given the addion of an extra inner detector pixel layer during the long shutdown of the LHC in 2014-15. This results in a light jet rejection factor of about 380 and a charm rejection factor around 12 for a b-tagging efficiency of $70 \%$. In the zero- and two-lepton channels the vector boson was required to have a $p_{T}>150 \mathrm{GeV}$. Different analysis categories were defined according to the number of leptons in the final state, the number of jets in the final state (two or three for the zeroand one-lepton analysis, and two or more than two for the two-lepton analysis), and the transverse momentum of the vector boson (larger or smaller than $150 \mathrm{GeV}$ for the two-lepton analysis). A detailed description of the selection and the results in this channel can be found in [4].

After the selection, the dominant backgrounds are $Z+j e t s$ in the zero- and two-lepton channels, and top quark production and $W+$ jets in the one-lepton channel. Further background reduction is obtained using a boosted decision tree (BDT) that combines 17 different variables and is trained independently for the eight different analysis categories. The largest discrimination is obtained from the correlation of the invariant mass of the b-jet pairs and the separation of the two b-jets. Two new variables that were not used in previous analyses were included, corresponding to the

\footnotetext{
${ }^{1}$ Only electrons or muons are considered.
} 

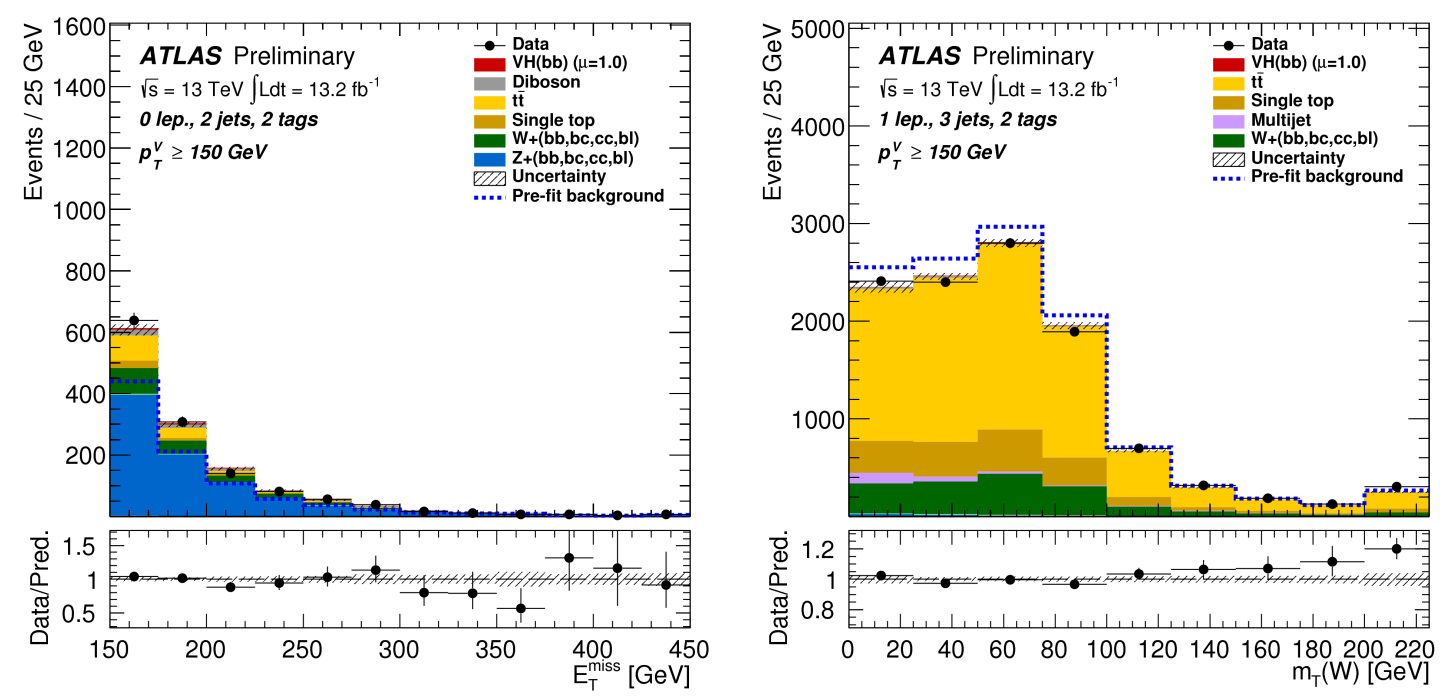

Figure 1: Missing transverse energy distribution in the zero-lepton channel (left), transverse mass of the $W$ boson in the one-lepton channel (right) for the VH Higgs boson search [4].

mass of the top quark in $t \bar{t}$ events and to the difference in pseudorapidity between the vector boson and the Higgs. They provide an improvement of $7 \%$ in the expected signal significance.

The signal strength, $\mu$, defined as the ratio of the observed cross section times branching ratio with respect to the SM expectation, is obtained from a profiled likelihood fit that takes into account all the analysis categories and uses the BDT discriminant as input. The dominant backgrounds are normalized in the fit. The distributions of the missing transverse energy in the zero-lepton channel, the transverse mass of the $W$ boson in the one-lepton channel and the BDT score in the two-lepton channels are shown in figure 1 (left and right) and 2 (left), respectively. The background normalizations are those resulting from the fit. The pre-fit background expectations are also shown. After the fit, there is good agreement between the data and the background expectations in all the three channels, taking into account the uncertainties.

The event yields as a function of the logarithm of the signal over background are presented in figure 2 (right), combining all the different analysis categories. The Higgs signal is expected to accumulate on the right-most bins. The data agrees well with the Standard Model background predictions, as shown on the data over background ratio in the lower panel. No clear excess of events with respect to the background expectations is seen.

The best fit signal strength is shown in figure 3 (left), for a $125 \mathrm{GeV}$ Higgs boson mass for the three different channels and for its combination. The largest signal strength is observed in the zero-lepton channel, $\mu_{0-\text { lept }}=0.47_{-0.69}^{+0.73}$, while the smallest is obtained for the two-lepton analysis $\left(\mu_{2-l e p t}=-0.24_{-0.84}^{+0.90}\right)$. In all the cases they are well below the SM expectations, although still compatible with them given the large uncertainties. The combined signal strength is $\mu=0.21_{-0.35}^{+0.36}(\text { stat })_{-0.36}^{+36}($ sys $)=0.21_{-0.50}^{+0.51}$. The observed (expected) $95 \%$ confidence level (CL) limit on the ratio of the cross section times branching ratio with respect to the SM expectation for $m_{H}=125 \mathrm{GeV}$ is $1.2\left(1.0_{-0.3}^{+0.4}\right)$, corresponding to an observed (expected) signal significance of 0.42 (1.94) standard deviations. The total systematic uncertainty is of the same size as the statis- 

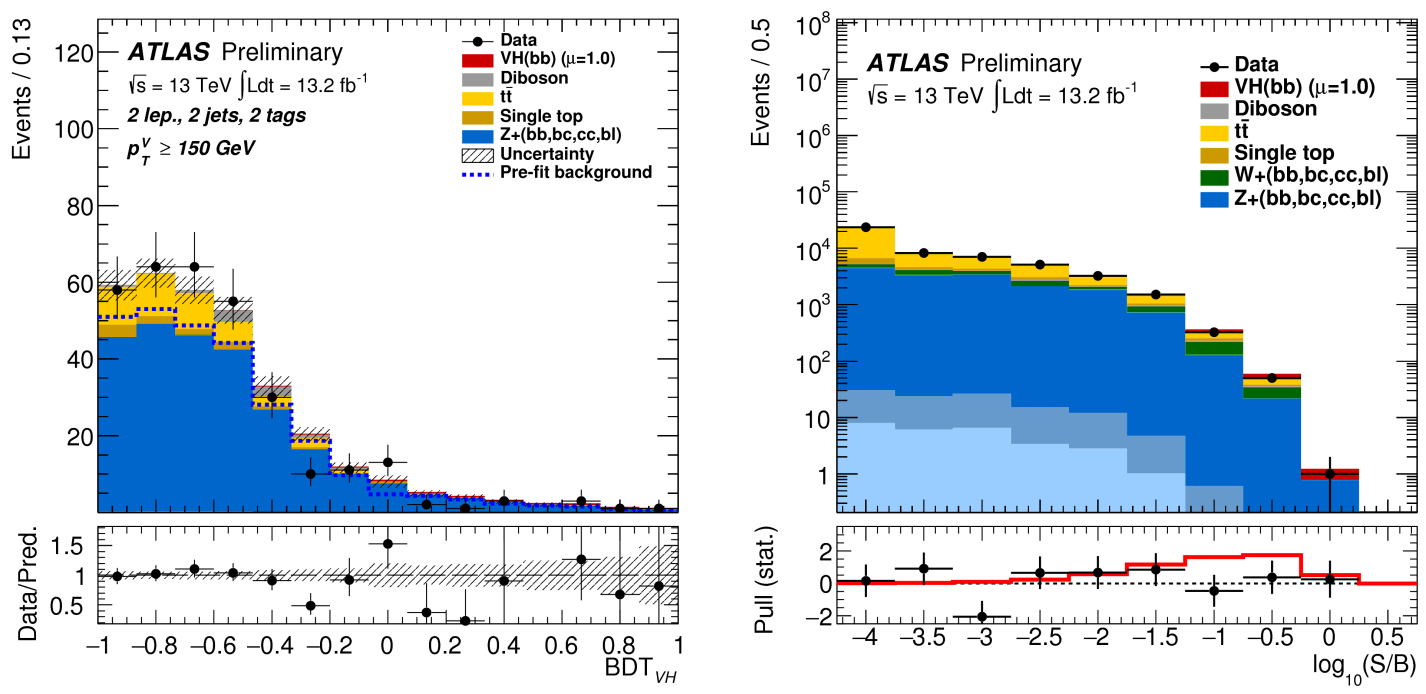

Figure 2: Left: BDT score in the two-lepton channel of the VH Higgs search. Right: Event yields as a function of $\log (\mathrm{S} / \mathrm{B})$ for data, background and Higgs boson signal with $m_{H}=125 \mathrm{GeV}$ for the combination of all channels in the VH Higgs search. Final-discriminant bins in all signal regions are combined into bins of $\log (\mathrm{S} / \mathrm{B})$. The signal $\mathrm{S}$ and background B yields are the expected and fitted values, respectively. The pull of the data with respect to the background-only prediction is shown without systematic uncertainties [4].

tical uncertainty, and is dominated by the background normalization and modelling and b-tagging uncertainties.

The fit was cross checked by searching for the standard model di-boson signal, $W Z+Z Z$, where the $Z$ boson decays to b-quark pairs. In this case, the observed signal significance is $3.2 \sigma$ with a signal strength of $\mu_{d i-b o s o n}=0.91 \pm 0.17(\text { stat })_{-0.23}^{+0.32}($ sys $)$, showing the validity of the fit procedure.

\section{Search for $H \rightarrow b b$ in vector boson fusion plus photon production}

This channel is characterized by a high- $\mathrm{p}_{\mathrm{T}}$ photon, two b-jets from the Higgs decay and two high- $\mathrm{p}_{\mathrm{T}}$ forward-backwards jets with large invariant mass and large rapidity separation, that is the typical signature of VBF production. Events are pre-selected with a dedicated trigger that requires a photon and four jets [5]. Offline, the event is required to have a good isolated photon, two b-tagged jets with an invariant mass $m_{b b}$ larger than $80 \mathrm{GeV}$ and two extra jets with invariant mass $m_{j j}>800 \mathrm{GeV}$. The dominant backgrounds at this point are $W$ or $Z$ production with a photon and additional jets, top-quark production with photons and jets, and multi-jet production of non-resonant heavy flavour jets in association with a photon. To further improve the background rejection, a BDT discriminant is built using seven different variables that are not correlated with $m_{b b}$, such as the distance between the jet and the photon, the invariant mass of the VBF jets or the centrality of the photon. The distribution of the BDT response for the Higgs signal and the simulated backgrounds is shown in figure 3 (right). Three different regions are defined using the BDT score: low $(-1.0<$ BDT $<-0.1)$, medium $(-0.1<$ BDT $<0.1)$ and high $(0.1<$ BDT $<1.0)$. The invariant mass distribution of the b-jet pairs for the low and high $B D T$ score regions are shown 
in figure 4. The expected SM $H \rightarrow b \bar{b}$ distribution multiplied by a factor ten is shown as a solid line. The data agrees well with the background expectations as shown in the lower pannels of the figure.
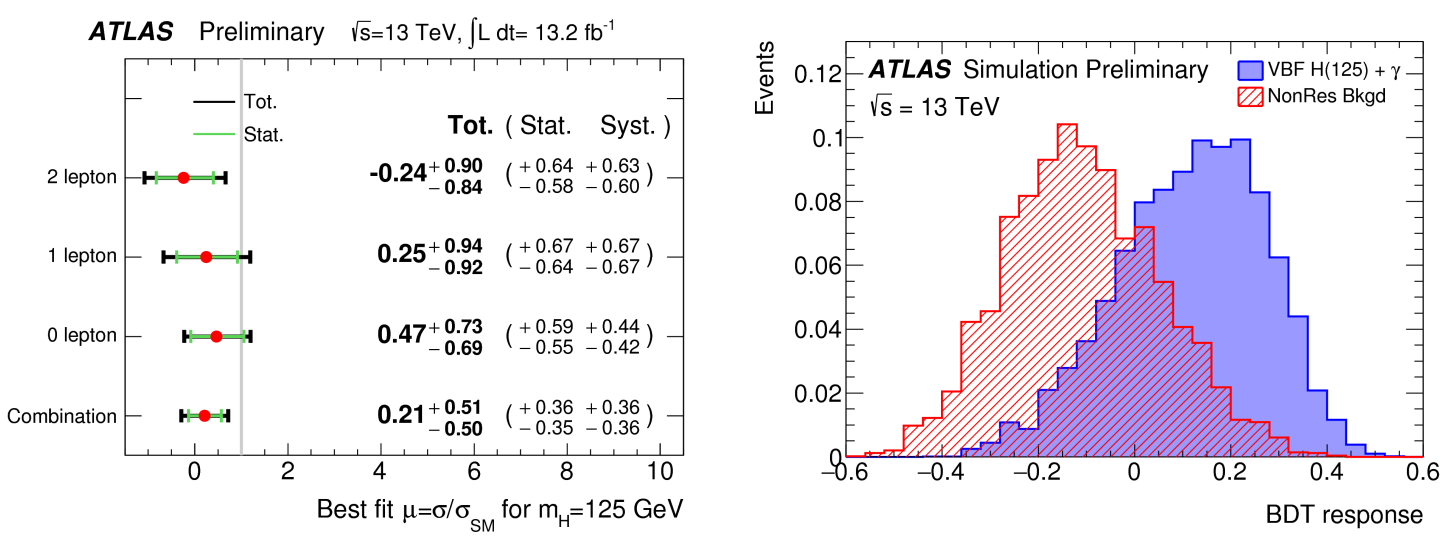

Figure 3: Left: the fitted values of the Higgs boson signal-strength parameter, $\mu$, for $m_{H}=125 \mathrm{GeV}$ for the zero-, one- and two-lepton $\mathrm{VH}$ channels [4]. Right: BDT response in the $\mathrm{VBF}+\gamma$ Higgs channel for the signal (in blue) and for the background (red) [5].
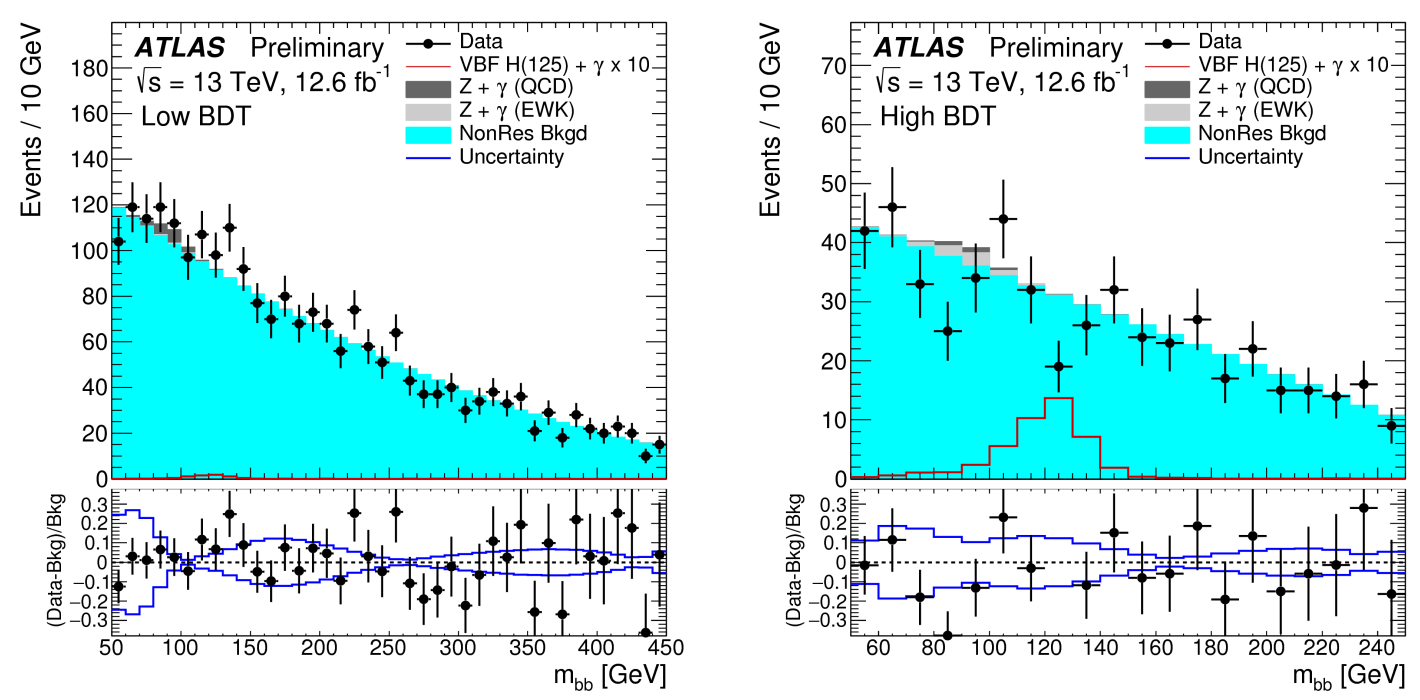

Figure 4: Invariant mass distribution of the b-jet pairs in the region with low (left) and high (right) BDT score [5] .

The di-b-jet invariant mass distributions of events falling in these three categories are fitted using a profiled likelihood fit, where the non-resonant background is represented by a smoothlyfalling polynomial distribution and the signal with a Crystal Ball function. The fit is tested by searching for the SM $Z \rightarrow b \bar{b}$ signal produced in VBF in association with a photon. The expected significance in the $Z(\rightarrow b \bar{b})+\gamma j j$ search was 1.3 while the observed value was 0.4 . The fitted signal strength was $\mu_{Z(\rightarrow b \bar{b})+\gamma j j}=0.3 \pm 0.8$. For the Higgs boson search, the expected 95\% CL limit was $6_{-1.7}^{+2.3}$ times the SM expectation, while the observed one was 4.0 times the SM prediction. The 
dominant systematic uncertainties come from the background estimation, theoretical uncertainties in the Higgs $+\gamma$ acceptance and cross section and jet energy scale.

\section{Summary and conclusions}

The search for the Higgs boson decaying to b-quark pairs is essential to probe the nature of the Higgs boson and test the predictions of the Standard Model, since it can probe the Yukawa coupling to down-type quarks and can be used to constrain the total width of the Higgs.

ATLAS has searched for the Higgs boson decaying to b-quark pairs in the associated production with a vector boson $\left(W\right.$ or $Z$ ) and in vector boson fusion with an extra high- $\mathrm{p}_{\mathrm{T}}$ photon, using $\sim 13 \mathrm{fb}^{-1}$ of $13 \mathrm{TeV}$ pp collisions. For the VH associated production, the expected significance is 1.92 , while the observed one is 0.42 , corresponding to a signal strengh of $\mu_{H, V H(H \rightarrow b . \bar{b})}=0.21_{-0.50}^{+0.51}$. In the $\mathrm{VBF}+\gamma$ channel, the expected $95 \% \mathrm{CL}$ limit is $6_{-1.7}^{+2.3}$ times the SM expectation, while the observed one is 4.0. The later is the first world result presented in this channel.

\section{Acknowlegdements}

The author acknowledges the support of the grant CERN/FIS-NUC/0005/2015, the program Investigador FCT, POPH, EU and FCT, Portugal.

\section{References}

[1] ATLAS Collaboration, Observation of a new particle in the search for the Standard Model Higgs boson with the ATLAS detector at the LHC, Phys. Lett. B 716 (2012) 1.

CMS Collaboration,Observation of a new boson at a mass of $125 \mathrm{GeV}$ with the CMS experiment at the LHC, Phys. Lett. B 716 (2012) 30.

[2] ATLAS, CMS collaborations, Measurements of the Higgs boson production and decay rates and constraints on its couplings from a combined ATLAS and CMS analysis of the LHC pp collision data at $\sqrt{s}=7$ and 8 TeV, J. High Energ. Phys. (2016) 2016: 45.

[3] ATLAS Collaboration, The ATLAS Experiment at the CERN Large Hadron Collider, JINST 3 (2008) S08003.

[4] ATLAS Collaboration, Search for the Standard Model Higgs boson produced in association with a vector boson and decaying to a b $\bar{b}$ pair in pp collisions at $\sqrt{s}=13 \mathrm{TeV}$ using the ATLAS detector, ATLAS-CONF-2016-091, https://atlas.web.cern.ch/Atlas/GROUPS/PHYSICS/CONFNOTES/ATLAS-CONF-2016-091/.

[5] ATLAS Collaboration, Search for Higgs boson production via weak boson fusion and decaying to $b \bar{b}$ in association with a high-energy photon using the ATLAS detector, ATLAS-CONF-2016-063, https://atlas.web.cern.ch/Atlas/GROUPS/PHYSICS/CONFNOTES/ATLAS-CONF-2016-063/. 